\title{
First-principles theoretical evaluation of crystalline zirconia and hafnia as gate oxides for Si microelectronics
}

\author{
Vincenzo Fiorentini and Gianluca Gulleri \\ INFM and Dipartimento di Fisica, Università di Cagliari, \\ Cittadella Universitaria, I-09042 Monserrato (CA), Italy
}

(Dated: October 25, 2018)

\begin{abstract}
Parameters determining the performance of the crystalline oxides zirconia $\left(\mathrm{ZrO}_{2}\right)$ and hafnia $\left(\mathrm{HfO}_{2}\right)$ as gate insulators in nanometric Si electronics are estimated via ab initio calculations of the energetics, dielectric properties, and band alignment of bulk and thin-film oxides on Si (001). With their large dielectric constants, stable and low-formation-energy interfaces, large valence offsets, and reasonable (though not optimal) conduction offsets (electron injection barriers), zirconia and hafnia appear to have a considerable potential as gate oxides for Si electronics.

PACS numbers: 68.35.-p, 77.22.-d, 85.30.-z, 61.66.-p
\end{abstract}

The performance needs of modern information technology are forcing Si-based ultra-large-scale-integrated (ULSI) devices into the domain of nanometric dimensions. This downscaling implies, among others, the effective continuing reduction of the physical thickness of insulating gate oxide layers in CMOS (Complementary Metal-Oxide-Semiconductor) devices. Amorphous $\mathrm{SiO}_{2}$, the natural oxide of Si technology, is now nearing its fundamental size limits, with physical thicknesses currently down to 2 unit cells [1]. This leads to uncomfortably large $\left(>1 \mathrm{~A} / \mathrm{cm}^{2}\right)$ leakage currents and increased failure probabilities. The main reason for the strong reduction of gate-oxide thickness in device downscaling is the need for increasing capacitances in the CMOS conducting channel. In a CMOS, the gate oxide layer dominates the series capacitance of the channel. An increase in capacitance can be obtained reducing the dielectric thickness $d / \varepsilon$ of the oxide layer, having physical thickness $d$ and relative dielectric constant $\varepsilon$. Given its small dielectric constant, it is understandable that $\mathrm{SiO}_{2}$ as a gate oxide has emerged as one of the key bottlenecks in device donwscaling [1, 2.

It thus appears that, if Moore's law [3] on ULSI circuit component density - and hence circuit performance - is to remain valid in the next decade, a replacement will have to be found for silica as a gate insulator. The basic selection criteria for such a replacement are $i$ ) larger dielectric constant ("high- $\kappa$ "), ii) interface band offsets to $\mathrm{Si}$ as large as or comparable to those of silica (especially the electron injection barrier), iii) epitaxy on Si energetically not too costly, $i v$ ) thermodynamical stability in contact with Si. In this work we address the expected performance, in terms of the above criteria, for the two important current candidates [1, 2, 4] hafnia $\left(\mathrm{HfO}_{2}\right)$ and zirconia $\left(\mathrm{ZrO}_{2}\right)$ through first-principles density-functional calculations of the structure, energetics, thermodynamical stability, dielectric constants, and band offsets of crystalline hafnia and zirconia thin films epitaxially grown on the (001) face of crystalline Si. We find stable and moderate-cost interfaces, large dielectric constants, and large band offsets, except for the electron injection barrier, estimated at $1 \mathrm{eV}$ at most, appreciably lower than the Si/silica barrier.

Our density functional theory calculations in the generalized gradient approximation [5] use the VASP code [6] and the ultrasoft [7] pseudopotentials provided therewith. Semicore states are treated as core for $\mathrm{Hf}$ and $\mathrm{Zr}$; test calculations done including the semicore as valence using the all-electron PAW [8] method as implemented in VASP 6] confirmed the pseudopotential results. Bulk optimizations were done in a 12-atom (conventional fcc or fct) cell, while the interfaces are simulated by (001)oriented oxide/Si superlattices contained in tetragonal cells of $c(2 \times 2)$ basal section, and in-plane lattice constant $a_{\mathrm{Si}}=5.461 \AA$, our theoretical value for bulk Si. Interface supercells contain around 50 atoms depending on the local interface structure, with 9 layers (18 atoms) for the Si region, and typically 11 layers (e.g. 24 oxygen and $10 \mathrm{Zr}$ atoms) for the oxide region. The plane-wave basis cutoff is $350 \mathrm{eV}$; for the $\mathrm{k}$-space summation we use $4 \times 4 \times 4$ meshes for the bulk and $4 \times 4 \times 1$ meshes for the $z$-elongated interface supercells.

Bulk and Si-epitaxial structure - Bulk hafnia and zirconia were studied in the fluorite, monoclinic, and Siepitaxial structures. The lattice parameters for $\mathrm{ZrO}_{2}$ are $a=5.10 \AA$ for fluorite, and $(a, b, c)=(5.186,5.255,5.351)$ $\AA$, off-normal angle $\theta=8.83^{\circ}$ for monoclinic. For $\mathrm{HfO}_{2}$, $a=5.06 \AA$ for fluorite, and $(a, b, c)=(5.108,5.175,5.280)$ $\AA$, off-normal angle $\theta=8.80^{\circ}$ for monoclinic. The latter phase is favored over fluorite by $0.115 \mathrm{eV} /$ formula unit for $\mathrm{ZrO}_{2}$ and by $0.248 \mathrm{eV} /$ formula unit for $\mathrm{HfO}_{2}$. The results agree with experiment and with recent calculations 19. 10, 11. The formation enthalpies $\Delta \mathrm{H}_{\mathrm{ox}}$ are -11.52 $\mathrm{eV}$ and -10.74 for hafnia and zirconia respectively (close to experiment, as usual using GGA) compared to -8.30 $\mathrm{eV}$ for silica: therefore both oxides are stable in contact with $\mathrm{Si}$ with respect to the decomposition into silica and metal. The same holds for the epitaxial phase discussed next, whose excess energy is only about $0.2 \mathrm{eV} /$ formula above the monoclinic. 
The tetragonal Si-epitaxial crystalline phase of each oxide was obtained imposing the in-plane lattice constant of $\mathrm{Si}$, and adjusting the axial ratio and internal coordinates in the 12 -atom conventional cells. The axial ratios $c / a_{\mathrm{Si}}$ are 0.92 for $\mathrm{ZrO}_{2}$ and 0.90 for $\mathrm{HfO}_{2}$. We verified by variable-cell damped dynamics [6] that this tetragonal bulk is stable against monoclinic distortions. The Siepitaxial configuration, depicted in Fig. 1 1 for $\mathrm{ZrO}_{2}$, may be viewed as a $z$-stacking of cation-anion bilayers alternatingly oriented at $90^{\circ}$ to each other, in which a) metal cations are disposed in dimerized (110)-like rows (cationcation distances within the rows 3.4 and $4.2 \AA$ compared to $3.86 \AA$ ideally), and $b$ ) oxygens quadruplets, originally square in fluorite, elongate to rhomboids along the (110) rows bending slightly sideways. The cation (anion) coordination decreases from 8 to 6 (from 4 to 3 ), in partial analogy to the monoclinic structure [10].

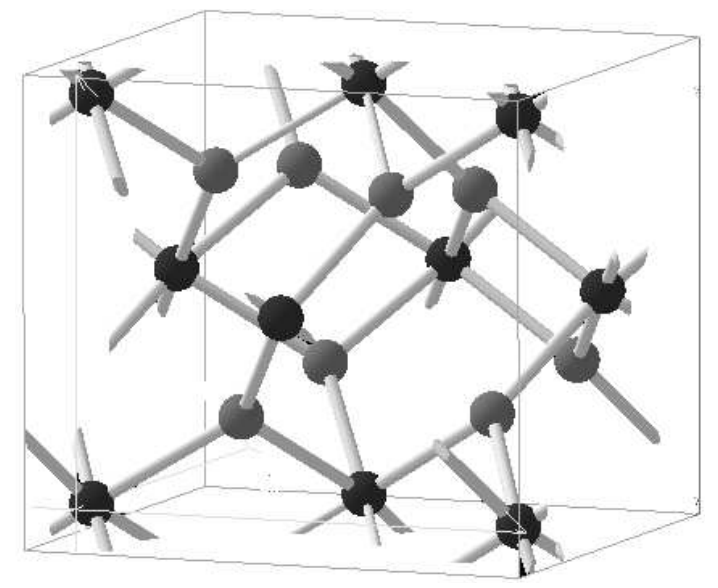

FIG. 1: Si-epitaxial structure of $\mathrm{ZrO}_{2}$. Grey (black) atoms: $\mathrm{O}(\mathrm{Zr})$.

The elastic energy $E_{\text {elastic }}^{\mathrm{epi}}$ of the Si-epi distorted bulk is $0.23 \mathrm{eV} /$ formula or $5.87 \mathrm{meV} / \AA^{3}$ for $\mathrm{ZrO}_{2}$, and 0.16 $\mathrm{eV} /$ formula or $4.37 \mathrm{meV} / \AA^{3}$ for $\mathrm{HfO}_{2}$ with respect to monoclinic bulk (i.e. both are slightly favored energetically over fluorite, whose occurrence is anyway barred by symmetry). While substantial, these energies are comparable to those of order $\sim 4 \mathrm{meV} / \AA^{3}$ involved (for much smaller strains) in nitride semiconductor epitaxy [12]. As we now discuss, the knowledge of the volume-specific epitaxial strain energy enables us to extract an area-specific interface energy, as well as to estimate the critical pseudomorphic growth thickness.

Interface energetics and offsets - Assuming a $c(2 \times 2)$ basal section, we investigated for both materials several local structures and terminations of oxide/Si (001) interfaces, e.g. Si/O, Si/metal, Si/metal-bilayer, mixed Si-metal layer/O, mixed Si-metal layer/O with $50 \%$ vacancies. The starting configuration of the oxide portion of the interface superlattices is assembled using the optimized Si-epi structure. The supercell length and atomic
TABLE I: Formation energies $\left(\mathrm{eV} / \AA^{2}\right)$ of, and valence and conduction band offsets (eV) at different Si (001)/oxide interfaces. The assumed growth conditions are indicated. The best offset/energetics combinations are displayed in underlined bold for metal-rich conditions, and bold for oxygen rich conditions. All GW corrections are included.

\begin{tabular}{c|c|ccc|ccr}
\hline \hline Material & $\rightarrow$ & \multicolumn{3}{|c|}{$\mathrm{HfO}_{2}$} & \multicolumn{3}{|c}{$\mathrm{ZrO}_{2}$} \\
\hline Interface $\downarrow$ & Growth & VBO & $\mathrm{CBO}$ & $\mathrm{E}_{\text {form }}$ & VBO & $\mathrm{CBO}$ & $\mathrm{E}_{\text {form }}$ \\
\hline $\mathrm{Si} / \mathrm{O}$ & O-rich & 4.14 & 0.47 & -0.16 & 4.08 & $\mathbf{0 . 7 2}$ & $-\mathbf{0 . 2 1}$ \\
$\mathrm{Si}-\mathrm{M} / \mathrm{O}$ & stoich & 4.40 & 0.19 & 0.17 & 4.18 & 0.62 & 0.12 \\
$\mathrm{Si} / \mathrm{M}$ & M-rich & 3.96 & 0.65 & 0.12 & 4.72 & 0.08 & 0.07 \\
$\mathrm{Si}-\mathrm{M} / \mathrm{O}$ vac & M-rich & & & & 3.91 & $\mathbf{0 . 8 9}$ & $\underline{\mathbf{0 . 1 5}}$ \\
$\mathrm{Si} / \mathrm{O}$ vac & stoich & 4.62 & -0.01 & 0.22 & 3.70 & 1.10 & 0.13 \\
\hline \hline
\end{tabular}

positions are then reoptimized: the axial ratio remains unchanged, and relaxations occur only in the first two interface-neigboring layers. The interface energy can be expressed as the difference of the energies $E_{\mathrm{SL}}$ of the interface cell, and $E_{\text {bulk }}$ of the corresponding bulk components, as

$$
\begin{aligned}
E_{\text {form }} & =\frac{1}{2 A}\left[E_{\mathrm{SL}}-E_{\mathrm{bulk}}\right]=\frac{1}{2 A}\left[\left(2 A \delta+n_{\mathrm{Si}} V_{\mathrm{Si}} E_{\mathrm{Si}}+\right.\right. \\
& \left.\left.+n_{\mathrm{ox}} V_{\mathrm{ox}} E_{\mathrm{ox}}\right)-\left(n_{\mathrm{Si}} V_{\mathrm{Si}} E_{\mathrm{Si}}+n_{\mathrm{ox}} V_{\mathrm{ox}}^{\prime} E_{\mathrm{ox}}^{\prime}\right)\right]=\delta
\end{aligned}
$$

with $n$ the number of bulk units, $V, V^{\prime}$ and $E, E^{\prime}$ the corresponding volumes and energies per unit volume, $A$ the basal superlattice area. The formation energy per unit area, $\delta$, can be extracted unambiguously if the oxide bulk energy is calculated in the same strain state as in the superlattice (Si remains unstrained), as in that case all volume-dependent terms drop off. Any other choice of the bulk energies inserts a volume dependence in the interface energy [12].

The interface cell may be stoichiometric, metal- or oxygen-deficient depending on its local structure. Its formation energy will therefore depend on growth conditions, metal-rich ones favoring oxygen deficit, and Orich favoring oxygen excess. Theoretically, this is described by fixing the chemical potentials of the constituents. Here, only one potential - e.g. oxygen's is independent: $\mu_{\mathrm{O}}=\mu_{\mathrm{O}_{2}} / 2$ means O-rich conditions, and $\mu_{\mathrm{O}}=\mu_{\mathrm{O}_{2}} / 2+\Delta \mathrm{H}_{\mathrm{ox}} / 2$ metal-rich ones.

The formation energies of the various interfaces are listed in Table I. The standard Si-O interface is favored in O-rich growth conditions. In metal-rich conditions, the preferred structure is the mixed Si-metal to $50 \%$ vacant oxygen layer interface depicted in Fig. 2, which remarkably is the same as was recently obtained [13] in all-electron ab-initio molecular dynamics simulations of metal deposition on, and oxidation of, $\mathrm{Si}$ (001). Notably, the two favored interfaces have large negative formation energies (referred, we remind, to the pre-strained bulk). This energetic gain in interface formation will be counterbalanced by the excess energy of the film's upper surface, and by the build-up of epitaxial elastic energy in 
the growing layer. An estimate of the critical thickness $t_{c}$ for pseudomorphic growth over an area $A$ then results from

$$
A E_{\text {form }}+A t_{c} E_{\text {elastic }}^{\mathrm{epi}}+A E_{\text {surf }}=0,
$$

using which we predict that crystalline zirconia and hafnia thin films should grow pseudomorphically on $\mathrm{Si}(001)$ : indeed, using our calculated values for, e.g., zirconia, and the GGA surface-energy estimate for the tetragonal phase $E_{\text {surf }} \simeq 0.05 \mathrm{eV} / \AA^{2}$ 14, we obtain $t_{c} \sim 18 \AA$ and $27 \AA$ for metal- and oxygen-rich conditions respectively. The poly-Si gate, forming a $\mathrm{Si} /$ oxide interface in the place of a free oxide surface, should further stabilize the structure.

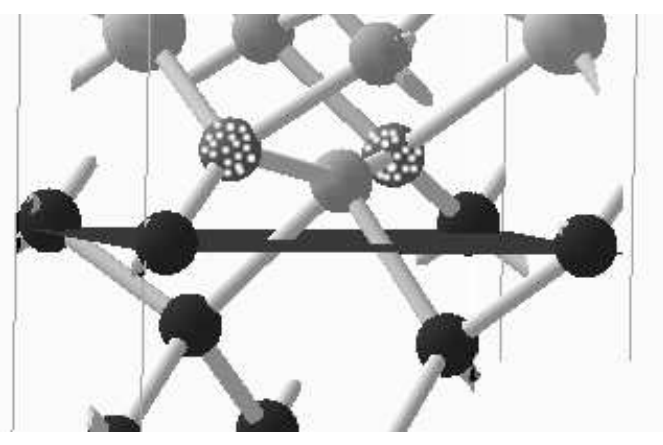

FIG. 2: The [mixed metal-Si]/[O 50\% vacant] interface (black: $\mathrm{Si}$; grey: metal; white-dotted: O) .

The interface band offsets are evaluated for each interface using the standard 'bulk-plus-lineup' procedure $[15$, expressing the valence offset (VBO) as the sum of the interface potential lineup and the valence-band-top differences of the separately-considered bulks. The conduction band offsets, hence the electron injection barriers, is estimated as $\mathrm{CBO}=\mathrm{E}_{\text {gap }}^{\text {oxide }}-\mathrm{E}_{\text {gap }}^{\mathrm{Si}}-\mathrm{VBO}$. The gap of $\mathrm{Si}$ is taken to be $1.1 \mathrm{eV}$; for both oxides, we use our GGA gaps corrected with the GW data of Ref. [17 for $\mathrm{ZrO}_{2}$, namely $5.9 \mathrm{eV}$ and $5.7 \mathrm{eV}$ for zirconia and hafnia respectively. These values are close to experiment for hafnia, and near the bottom of the (large) experimental range for zirconia. We neglect spin-orbit corrections, which should be well below $\pm 0.1 \mathrm{eV}$ as the valence states are oxygenderived. We do include, instead, the quasiparticle corrections to the bulk valence-band edges at the GW level: this is essential since these corrections are of order $\sim 1$ $\mathrm{eV}$ in oxides compared to typical $\sim 0.1 \mathrm{eV}$ in semiconductors. We apply to the VBOs an overall correction of $-1.08 \mathrm{eV}$, resulting from the $-0.15 \mathrm{eV}$ correction [16] for $\mathrm{Si}$ and the $-1.23 \mathrm{eV}$ correction 17] for $\mathrm{ZrO}_{2}$. Using the latter for both oxides introduces some uncertainty in the $\mathrm{HfO}_{2}$ results, but unfortunately no GW data are currently available for hafnia.

In Table 1 we report the predicted VBOs and CBOs. Qualitatively, VBOs cluster around $4 \mathrm{eV}$, with appreciable structure dependence, and $\mathrm{CBOs}$ are in the range 0 to $1 \mathrm{eV}$. Interestingly, for zirconia the energy-wise most favorable structures have some of the largest conduction offsets. The high-end CBOs, $\sim 1 \mathrm{eV}$, are smaller than, but comparable to, the $1.4-1.5 \mathrm{eV}$ estimates by Robertson 18], who used a simple charge-neutrality-level model at the empirical tight-binding model.

Dielectric constants - The lattice contribution to the dielectric tensor has been calculated for both oxides in the fluorite, monoclinic, and Si-epitaxial structures. We used a standard formalism to evaluate the zero-frequency dielectric constant [10] via the frequencies of zone-center IR-active modes and the transverse dynamical charges. The vibrational modes are calculated diagonalizing the zone-center dynamical matrix $-\partial F_{i}^{\alpha} / \partial u_{j}^{\beta}$, obtained differentiating by centered finite-differences (with displacements of $0.1 \AA$ ) the Hellmann-Feynman force component $\alpha$ on atom $i$ with respect to the displacement of atom $j$ along direction $\beta$. The dynamical charges are likewise obtained by finite-difference differentiation of the Berryphase 19] polarization with respect to atomic displacements (of typically $0.05 \AA$ ).

TABLE II: Lattice dielectric tensor for fluorite, monoclinic, and Si-epitaxial $\mathrm{XO}_{2}$ (the small off-diagonal elements for the monoclinic are not displayed for clarity), calculated using the dynamical charge tensor of fluorite. $\varepsilon_{\text {lat }}^{\text {ave }}$ is the orientational average measured by series capacitance in polycristalline layers, and obtained as $3 / \varepsilon^{\text {ave }}=1 / \varepsilon^{x x}+1 / \varepsilon^{y y}+1 / \varepsilon^{z z}$,

\begin{tabular}{lccc|c}
\hline \hline & $\varepsilon_{\text {lat }}^{x x}$ & $\varepsilon_{\text {lat }}^{y y}$ & $\varepsilon_{\text {lat }}^{z z}$ & $\varepsilon_{\text {lat }}^{\text {ave }}$ \\
\hline $\mathrm{HfO}_{2}$ fluorite & 27.8 & 27.8 & 27.8 & 27.8 \\
$\mathrm{HfO}_{2}$ monoclinic & 17.5 & 15.7 & 12.4 & 14.9 \\
$\mathrm{HfO}_{2}$ Si-epi & 27.6 & 18.6 & 24.5 & 22.9 \\
\hline $\mathrm{ZrO}_{2}$ fluorite & 30.5 & 30.5 & 30.5 & 30.5 \\
$\mathrm{ZrO}_{2}$ monoclinic & 24.7 & 18.3 & 14.6 & 18.4 \\
$\mathrm{ZrO}_{2}$ Si-epi & 22.5 & 71.5 & 44.9 & 37.0 \\
\hline \hline
\end{tabular}

Since the epi-oxides were optimized without constraints, they have no symmetry of practical use. The calculation of the full dynamical charge tensor for all atoms in the complex epitaxial (as well as the monoclinic) structure is thus rather demanding, and currently in progress. In Table II we give estimates of the diagonal elements of the lattice dielectric tensor obtained using the dynamical charge tensor of the fluorite phase, which is diagonal and isotropic, and calculated to be $Z_{\mathrm{Hf}}^{*}=5.20$ and $Z_{\mathrm{O}}^{*}=-2.60$ for $\mathrm{HfO}_{2}, Z_{\mathrm{Zr}}^{*}=5.50$ and $Z_{\mathrm{O}}^{*}=-2.75$ for $\mathrm{ZrO}_{2}$. Of course, smaller dynamical charges such as found in monoclinic phases 10, 11] will decrease the dielectric constant, especially the $z z$ component. Using the monoclinic cation charge tensors of Refs. [10, 11] and imposing the Friedel sum rule to obtain an average anion charge tensor, we estimated $\varepsilon_{z z}$ to be 9.9 and 11.9 in monoclinic $\mathrm{HfO}_{2}$ and $\mathrm{ZrO}_{2}$ respectively, in fair agreement with previous results. Along with our fluorite values, also in good agreement with previous calculations, this gives us confidence on the reliability of our procedure. 


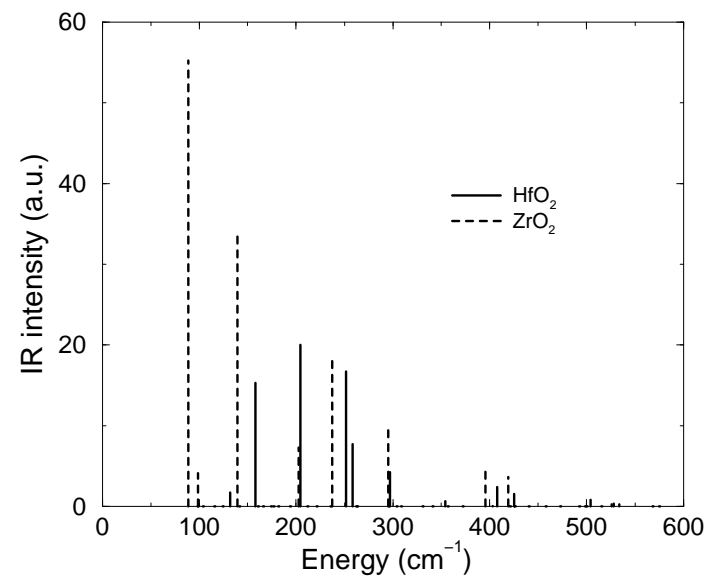

FIG. 3: Orientationally averaged IR intensity spectrum (mode dielectric constants) of Si-epitaxial $\mathrm{HfO}_{2}$ (solid) and $\mathrm{ZrO}_{2}$ (dashed).

With reference to Table II, for hafnia we find a reduction in dielectric constant compared to fluorite both in the Si-epi and monoclinic phases, though the latter is rather more dramatic, with a more than twofold decrease, in agreement with previous calculations 111. For zirconia, we also find a similar, approximately twofold reduction of the monoclinic dielectric tensor compared to fluorite; notably, though, a drastic enhancement is found in the Si-epitaxial phase. This results from the large IR intensity of modes at about $90 \mathrm{~cm}^{-1}$ to $140 \mathrm{~cm}^{-1}$, as can be seen in Fig.3, which reports the mode dielectric constants 10] for both materials in the Si-epi phase. The two lower-energy modes for zirconia (dashed lines) contribute mostly to the $y y$ component, the third to the $z z$ component. The pronounced softness of Si-epi zirconia is presumably due to the backfolding of zone-border $(X$ point) modes.

We carefully checked against artifacts by accurately reoptimizing structures and repeating phonon calculations for different displacements. We are confident in our procedure also in view of the results for the other phases. The single zone-center IR-active mode of fluorite is $\omega=230 \mathrm{~cm}^{-1}$ for $\mathrm{HfO}_{2}$ and $\omega=258 \mathrm{~cm}^{-1}$ for $\mathrm{ZrO}_{2}$; for the latter this agrees with recent predictions [9, 10], for the former the frequency is $20 \%$ lower than in Ref. 11 . We checked that the same results are obtained (within $0.5 \%$ for the lattice constant and $2 \%$ for the frequency) with the all-electron PAW method with valence semicore [6, 8]. The details of the vibrational spectrum of the epi and monoclinic phases will be reported elsewhere, but we note in passing that the results for the monoclinic are close to previous reports [11].

In conclusion, the picture of zirconia and hafnia as Sigate oxides as it emerges from this work is rather encouraging, certainly so from the dielectric and epitaxy- energetic standpoints. The results on the electron injection barriers are partly disappointing, as the electron injection barrier is much smaller that at silica/Si interfaces. While insufficient for hot electrons, the barrier should be still acceptable for standard two-dimensional inversion layers, whose energy levels are at about 100 meV above the interface triangular-well bottom [20].

We thank P. Blöchl and C. Först for communicating their surface results for Zr-O coadsorption on Si (001) prior to publication. We are grateful to A. Pasquarello and P. Pavone for helpful suggestions. This work was supported in part by the European Union (INVEST project), and by the Parallel Supercomputing Initiative of Istituto Nazionale per la Fisica della Materia.

[1] H. R. Huff, J. Electrochem. Soc. 149, S35 (2002); S. Thompson, P. Packan, and M. Bohr, Intel Techn. J. Q398, p.1 (2001).

[2] D. A. Buchanan, IBM J. Res. Devel. 43, 245 (1999); G. D. Wilk, R. M. Wallace, and J. M. Anthony, J. Appl. Phys. 89, 5243 (2001).

[3] G. E. Moore, Electronics 83, 114 (1965); SPIE 2438, 2-17 (1995).

[4] Y. Kim et al., IEEE Int. Electr. Dev. Meeting Techn. Dig. (Cat. No.01CH37224), p.20.2.1-4 (2001).

[5] J. P. Perdew, in Electronic Structure of Solids, P. Ziesche and H. Eschrig eds. (Akademie-Verlag, Berlin 1991), p.11.

[6] G. Kresse and J. Furthmüller, Comput. Mater. Sci. 6, 15 (1996); Phys. Rev. B 54, 11169 (1996); G. Kresse and D. Joubert, ibid. 59, 1758 (1999).

[7] D. Vanderbilt, Phys. Rev. B 41, 7892 (1990).

[8] P. E. Blöchl, Phys. Rev. B 50, 17953 (1994).

[9] G.-M. Rignanese, F. Detraux, X. Gonze, and A. Pasquarello, Phys. Rev. B 64, 134301 (2001)

[10] X. Zhao and D. Vanderbilt, Phys. Rev. B 65, 075105 (2002).

[11] X. Zhao and D. Vanderbilt, Phys. Rev. B 65, 233106 (2002).

[12] F. Bernardini and V. Fiorentini, Phys. Rev. B 57, R9427 (1998).

[13] P. Blöchl and C. Först, to be published.

[14] A. Christensen and E. A. Carter, Phys. Rev. B 62, 16968 (2000).

[15] M. Peressi, N. Binggeli, and A. Baldereschi, J. Phys. D 31, 1273 (1998).

[16] X. Zhu and S. G. Louie, Phys. Rev. B 43, 14142 (1991).

[17] B. Kralik, E. K. Chang, and S. G. Louie, Phys. Rev. B 57, 7027 (1998).

[18] J. Robertson, J. Vac. Sci. Technol. B 18, 1785 (2000).

[19] R. D. King-Smith and D. Vanderbilt, Phys. Rev. B 47, 1651 (1992); VASP implementation by Martijn Marsman.

[20] T. Ando, A. B. Fowler, and F. Stern, Rev. Mod. Phys. 54, 437 (1982). 